Mathematical Modelling and AnAlysis

Volume 17 Number 3, June 2012, 293-308

http://dx.doi.org/10.3846/13926292.2012.685497

(c) Vilnius Gediminas Technical University, 2012
Publisher: Taylor\&Francis and VGTU

http://www.tandfonline.com/TMMA

Print ISSN: 1392-6292

Online ISSN: 1648-3510

\title{
Optimization-Based Search for Nordsieck Methods of High Order with Quadratic Stability Polynomials*
}

\section{Angelamaria Cardone ${ }^{a}$, Zdzislaw Jackiewicz ${ }^{b, c}$ and Hans Mittelmann ${ }^{b}$}

${ }^{a}$ Università degli Studi di Salerno

via Ponte Don Melillo, I-84084 Fisciano (SA), Italy

${ }^{b}$ Arizona State University

85287 Tempe, Arizona

${ }^{c}$ AGH University of Science and Technology

Kraków, Poland

E-mail: ancardone@unisa.it

E-mail(corresp.): jackiewi@math.la.asu.edu

E-mail: mittelmann@math.asu.edu

Received September 23, 2011; revised February 20, 2012; published online June 1, 2012

\begin{abstract}
We describe the search for explicit general linear methods in Nordsieck form for which the stability function has only two nonzero roots. This search is based on state-of-the-art optimization software. Examples of methods found in this way are given for order $p=5, p=6$, and $p=7$.
\end{abstract}

Keywords: general linear methods, Nordsieck methods, quadratic stability, least-squares minimization.

AMS Subject Classification: 65L05; 65L20; 65K05.

\section{Introduction}

We describe the search for some class of explicit general linear methods (GLMs) of high order for the numerical solution of ordinary differential equations (ODEs). The construction of such methods is a highly nontrivial task and powerful minimization software is required to obtain methods of high accuracy with desirable stability properties. In [5] we constructed diagonally implicit multistage integration methods (DIMSIMs) up to order $p=4$ using symbolic manipulations packages such as Mathematica and Maple and subroutines based on continuation methods from PITCON [26, 27], ALCON [1, 2], and

\footnotetext{
* The work of the second author was partially supported by the National Science Foundation under grant NSF DMS-0509597.
} 
HOMPACK [29]. In [8] we have used the subroutines Imdif.f and Imder.f from MINPACK which minimize the sum of squares by a modification of the Levenberg-Marquardt algorithm [24] to construct the class of diagonally implicit multistage integration methods (DIMSIMs) of order $p$ and stage order $q=p$ for $p=5$ and $p=6$. In the papers [10] and [20] we have used more efficient optimization algorithms based on variable-model trust-region leastsquares algorithms implemented in the improved version of NL2SOL [16, 17] to construct explicit and implicit DIMSIMs of order $p=7$ and $p=8$. In [14] and [23] we have constructed two-step Runge-Kutta (TSRK) methods of low and moderate orders with large regions of absolute stability using fminsearch from Matlab [18]. These methods were introduced in [21, 22, 28]. The implicit TSRK methods of order up to $p=6$ were constructed in [3] and up to order $p=8$ in a recent paper [15]. In [13] we have described a search for a class of Nordsieck methods with $s$ internal stages, $r=s$ external stages of order $p=s-1$ and stage order $q=p$ and obtained methods with large regions of absolute stability up to order $p=4$. In this search we have used the approach based on minimizing the sum of squares of the difference between the coefficients of the stability function of the method and the coefficients of a given quadratic polynomial with a large area of stability. In this search we utilized fminsearch from Matlab. The obtained accuracy was sufficient only up to the order $p=3$ and had to be improved for methods of order $p=4$ by solving the system of nonlinear equations for the desired coefficients of stability function using fsolve from Matlab. For higher orders $(p \geq 5)$ the approach based on using fminsearch and fsolve failed to give sufficient accuracy and more powerful minimization software is required. In this paper we describe the construction of Nordsieck methods of order $p=5, p=6$ and $p=7$ using state-of-the-art optimization software.

The organization of this paper is as follows. In Section 2 we introduce the class of Nordsieck methods we are interested in and formulate order and stage order conditions. We also review representation formulas for some coefficients matrices of these methods. In Section 3 we describe the construction of quadratic polynomials of high order and large regions of stability. This construction is based on maximizing the area of the intersection of the region of absolute stability with the negative half plane. We also present examples of such optimal polynomials corresponding to $p=s-1=5, p=s-1=6$, and $p=s-1=7$. In Section 4 we describe the search for Nordsieck methods with quadratic stability. This search is based on minimizing the objective function for the sum of squares of the difference between the coefficients of the stability function of the method and coefficients of the optimal quadratic polynomials constructed in Section 3. In Section 5 we present examples of methods of order $p=5, p=6$ and $p=7$ computed using the approach described in Section 4 and compare their stability regions with the corresponding stability regions of approximation of the same order to the exponential function $\exp (z)$ and of the Nordsieck methods of the same order with so-called inherent Runge-Kutta stability (IRKS) constructed in [9]. Finally, in Section 6 some concluding remarks are given and plans for future research in this area are briefly outlined. 


\section{Nordsieck Methods}

For the numerical solution of initial value problem for ODEs

$$
\left\{\begin{array}{l}
y^{\prime}(t)=f(y(t)), \quad t \in\left[t_{0}, T\right], \\
y\left(t_{0}\right)=y_{0},
\end{array}\right.
$$

$f: \mathbb{R}^{m} \rightarrow \mathbb{R}^{m}$, we consider the class of Nordsieck methods defined by

$$
\begin{cases}Y_{i}^{[n]}=h \sum_{j=1}^{s} a_{i j} f\left(Y_{j}^{[n]}\right)+\sum_{j=1}^{r} u_{i j} z_{j}^{[n-1]}, & i=1,2 \ldots, s \\ z_{i}^{[n]}=h \sum_{j=1}^{s} b_{i j} f\left(Y_{j}^{[n]}\right)+\sum_{j=1}^{r} v_{i j} z_{j}^{[n-1]}, & i=1,2 \ldots, r\end{cases}
$$

$n=1,2, \ldots, N, t_{n}=t_{0}+n h, n=0,1, \ldots, N, N h=T-t_{0}$. Here, $Y_{i}^{[n]}$ is approximation of stage order $q$ to $y\left(t_{n}+c_{i} h\right)$ and $z_{i}^{[n]}$ is approximation of order $p$ to the component $h^{i-1} y^{(i-1)}\left(t_{n}\right)$ of the Nordsieck vector $z(t, h)$ defined by

$$
z(t, h)=\left[\begin{array}{llll}
y(t)^{T} & h y^{\prime}(t)^{T} & \cdots & h^{r-1} y^{(r-1)}(t)^{T}
\end{array}\right]^{T}
$$

and $y(t)$ is the solution to the problem (2.1). These methods are characterized by the abscissa vector $\mathbf{c}=\left[c_{1}, \ldots, c_{s}\right]^{T}$ and four coefficient matrices $\mathbf{A} \in \mathbb{R}^{s \times s}$, $\mathbf{U} \in \mathbb{R}^{s \times r}, \mathbf{B} \in \mathbb{R}^{r \times s}$, and $\mathbf{V} \in \mathbb{R}^{r \times r}$. It will be assumed throughout the paper that $r=s$ and $p=q=s-1$. It is known $[4,6,7,19]$ that the method $(2.2)$ has order $p$ and stage order $q=p$ if and only if

$$
\begin{aligned}
& e^{\mathbf{c} z}=z \mathbf{A} e^{\mathbf{c} z}+\mathbf{U} \phi(z)+O\left(z^{p+1}\right), \\
& e^{z} \phi(z)=z \mathbf{B} e^{\mathbf{c} z}+\mathbf{V} \phi(z)+O\left(z^{p+1}\right),
\end{aligned}
$$

where $\phi(z)=\left[\begin{array}{llll}1 & z & \cdots & z^{s-1}\end{array}\right]^{T}$. Put

$$
\mathbf{K}=\left[\begin{array}{ccccc}
0 & 1 & 0 & \cdots & 0 \\
0 & 0 & 1 & \cdots & 0 \\
\vdots & \vdots & \vdots & \ddots & \vdots \\
0 & 0 & 0 & \cdots & 1 \\
0 & 0 & 0 & \cdots & 0
\end{array}\right], \quad \mathbf{F}=\exp (\mathbf{K})=\left[\begin{array}{ccccc}
1 & 1 & \frac{1}{2 !} & \cdots & \frac{1}{p !} \\
0 & 1 & 1 & \cdots & \frac{1}{(p-1) !} \\
0 & 0 & 1 & \cdots & \frac{1}{(p-2) !} \\
\vdots & \vdots & \vdots & \ddots & \vdots \\
0 & 0 & 0 & \cdots & 1
\end{array}\right]
$$

Then it follows from stage order and order conditions (2.3) and (2.4) that the coefficient matrices $\mathbf{A}$ and $\mathbf{U}$ and $\mathbf{B}$ and $\mathbf{V}$ are related by

$$
\mathbf{U}=\mathbf{C}-\mathbf{A C K}, \quad \mathbf{V}=\mathbf{F}-\mathbf{B C K} .
$$

To search for methods with large regions of absolute stability it is more convenient to reformulate the relation between $\mathbf{V}$ and $\mathbf{B}$ in a different form. Put

$$
\mathbf{F}=\left[\begin{array}{llll}
f_{1} & f_{2} & \cdots & f_{s}
\end{array}\right], \quad \mathbf{B}=\left[\begin{array}{l}
\tilde{B} \mid \mathbf{b}_{s}
\end{array}\right], \quad \mathbf{V}=\left[\begin{array}{llll}
v_{1} & v_{2} & \cdots & v_{s}
\end{array}\right],
$$


where $f_{i}, v_{i} \in \mathbb{R}^{s}, i=1,2, \ldots, s, \tilde{B} \in \mathbb{R}^{s \times(s-1)}$, and $\mathbf{b}_{s} \in \mathbb{R}^{s}$. Let

$$
\mathbf{C}=\left[\begin{array}{lllll}
\mathbf{e} & \mathbf{c} & \frac{\mathbf{c}^{2}}{2 !} & \cdots & \frac{\mathbf{c}^{p}}{p !}
\end{array}\right] \in \mathbb{R}^{s \times s},
$$

and partition the matrix $\mathbf{C K}$ as follows

$$
\mathbf{C K}=\left[\begin{array}{c|c}
0 & \tilde{C} \\
\hline 0 & \tilde{c}
\end{array}\right]=\left[\begin{array}{c|cccc}
0 & 1 & c_{1} & \cdots & \frac{c_{1}^{p-1}}{(p-1) !} \\
0 & 1 & c_{2} & \cdots & \frac{c_{2}^{p-1}}{(p-1) !} \\
\vdots & \vdots & \vdots & \ddots & \vdots \\
0 & 1 & c_{s-1} & \cdots & \frac{c_{s-1}^{p-1}}{(p-1) !} \\
\hline 0 & 1 & c_{s} & \cdots & \frac{c_{s}^{p-1}}{(p-1) !}
\end{array}\right] .
$$

Then it was proved in [13] that the matrix $\tilde{B}$ has the representation

$$
\tilde{B}=\left(\left[\begin{array}{lll}
f_{2} & \cdots & f_{s}
\end{array}\right]-\left[\begin{array}{lll}
v_{2} & \cdots & v_{s}
\end{array}\right]-\mathbf{b}_{s} \tilde{c}\right) \tilde{C}^{-1}
$$

We next define the error constant $E$ of (2.2). Let the matrices $\mathbf{B}$ and $\mathbf{V}$ be partitioned as follows

$$
\mathbf{B}=\left[\begin{array}{c}
\mathbf{b}^{T} \\
\hline B
\end{array}\right], \quad \mathbf{V}=\left[\begin{array}{c|c}
1 & \mathbf{v}^{T} \\
\hline 0 & V
\end{array}\right]
$$

where $\mathbf{b} \in \mathbb{R}^{s}, B \in \mathbb{R}^{(s-1) \times s}, \mathbf{v} \in \mathbb{R}^{s-1}$, and $V \in \mathbb{R}^{(s-1) \times(s-1)}$. Then it was demonstrated in $[11,19]$ that if

$$
z_{1}^{[n-1]}=y\left(t_{n-1}\right), \quad z_{i}^{[n-1]}=h^{i-1} y^{(i-1)}\left(t_{n-1}\right)+O\left(h^{p+1}\right), \quad i=2,3, \ldots, s,
$$

then

$$
z_{1}^{[n]}=y\left(t_{n}\right)-h^{p+1} E y^{(p+1)}\left(t_{n}\right)+O\left(h^{p+2}\right),
$$

where the error constant $E$ is given by

$$
E=\frac{1}{(p+1) !}-\frac{\mathbf{b}^{T} \mathbf{c}^{p}}{p !}+\mathbf{v}^{T} \alpha
$$

Here,

$$
\alpha=(I-V)^{-1}\left(\mathbf{t}_{p}-\frac{B \mathbf{c}^{p}}{p !}\right) \quad \text { and } \quad \mathbf{t}_{p}=\left[\begin{array}{llll}
\frac{1}{p !} & \frac{1}{(p-1) !} & \cdots & 1
\end{array}\right]^{T} \in \mathbb{R}^{s-1} .
$$

In this paper we are interested in explicit Nordsieck methods, i.e., methods for which the coefficient matrix $\mathbf{A}$ is strictly lower triangular. We will also assume that the matrix $V$ appearing in $\mathbf{V}$ is strictly upper triangular. This implies that the method (2.2) is automatically zero-stable, i.e., the matrix $\mathbf{V}$ is power bounded. 
Applying the method (2.2) to the test equation $y^{\prime}=\xi y, t \geq 0, \xi \in \mathbb{C}$, we obtain $z^{[n]}=\mathbf{M}(z) z^{[n-1]}, z=h \xi, n=1,2, \ldots$, where the stability matrix $\mathbf{M}(z)$ is defined by

$$
\mathbf{M}(z)=\mathbf{V}+z \mathbf{B}(\mathbf{I}-z \mathbf{A})^{-1} \mathbf{U} .
$$

We also define the stability function $p(w, z)$ by

$$
p(w, z)=\operatorname{det}(w \mathbf{I}-\mathbf{M}(z)) .
$$

It is a big challenge to construct methods (2.2) which achieve a good balance between accuracy, i.e., the order $p$, stage order $q$, and the size of the error constant $E$, and stability as measured by the size of the region of absolute stability. In $[12,30,31]$ the concept of IRKS was introduced which guarantees that for explicit Nordsieck methods the stability function assumed the form

$$
p(w, z)=w^{s-1}(w-R(z, \eta))
$$

with $R(z, \eta)$ given by

$$
R(z, \eta)=1+z+\frac{z^{2}}{2}+\cdots+\frac{z^{s-1}}{(s-1) !}+\eta \frac{z^{s}}{s !}
$$

where $\eta$ is a free parameter. In $[9,19]$ this parameter was used to construct explicit Nordsieck methods (2.2) with small error constants $E$ and large regions of absolute stability. In [13] the concept of IRKS was relaxed to the concept of so-called inherent quadratic stability (IQS). These are some algebraic conditions imposed on the coefficient matrices of the method which guarantee that the stability function of (2.2) assumes the form

$$
p(w, z)=w^{s-2}\left(w^{2}-p_{s-1}(z) w+p_{s-2}(z)\right),
$$

with

$$
\begin{aligned}
& p_{s-1}(z)=1+p_{s-1,1} z+p_{s-1,2} z^{2}+\cdots+p_{s-1, s} z^{s}, \\
& p_{s-2}(z)=p_{s-2,1} z+p_{s-2,2} z^{2}+\cdots+p_{s-2, s} z^{s} .
\end{aligned}
$$

This approach leads to additional free parameters which can be used to construct methods with larger regions of absolute stability than those corresponding to methods with IRKS of the same order. In [13] we investigated also methods with quadratic stability (QS), i.e., methods for which stability polynomial $p(w, z)$ assumes the form $(2.9)-(2.10)$ but which do not necessarily satisfy IQS conditions. Examples of methods with IQS and QS up to order $p=4$ are presented in [13] and their regions compare favorably with methods which have IRKS.

In this paper we continue the search for high order $(p \geq 5)$ Nordsieck methods with QS. In this search we first find optimal quadratic polynomials $q_{\text {opt }}(w, z)$ with large regions of absolute stability and then solve a minimization problem to find methods for which the stability function takes the form $p(w, z)=w^{s-2} q_{o p t}(w, z)$. The construction of optimal quadratic polynomials 
which is based on maximizing the area of absolute stability of these polynomials is described in Section 3. This can be accomplished using fminsearch from Matlab. The construction of methods whose stability properties are determined by these optimal quadratic polynomials is described in Section 4. This construction utilizes the Levenberg-Marquardt method.

\section{Construction of Quadratic Polynomials with Large Re- gions of Stability}

In this section we describe the construction of quadratic polynomials corresponding to Nordsieck methods with $s=r$ and $p=q=s-1$. Such polynomials have the form

$$
q(w, z)=w^{2}-q_{s-1}(z) w+q_{s-2}(z)
$$

with

$$
\begin{aligned}
& q_{s-1}(z)=1+q_{s-1,1} z+q_{s-1,2} z^{2}+\cdots+q_{s-1, s} z^{s}, \\
& q_{s-2}(z)=q_{s-2,1} z+q_{s-2,2} z^{2}+\cdots+q_{s-2, s} z^{s},
\end{aligned}
$$

which satisfy the order conditions

$$
q\left(e^{z}, z\right)=e^{2 z}-q_{s-1}(z) e^{z}+q_{s-2}(z)=O\left(z^{s}\right) .
$$

These order conditions (3.1) lead to the system of $s-1$ linear equations for the coefficients of the polynomials $q_{s-1}(z)$ and $q_{s-2}(z)$. The resulting system of linear equations is then solved with respect to

$$
q_{s-1,1}, \ldots, q_{s-1,(s-1) / 2}, q_{s-2,1}, \ldots, q_{s-2,(s-1) / 2}
$$

if $s$ is odd, and with respect to $q_{s-1,1}, \ldots, q_{s-1, s / 2}, q_{s-2,1}, \ldots, q_{s-2, s / 2-1}$ if $s$ is even, and the remaining free parameters

$$
q_{\text {par }}=\left[\begin{array}{llllll}
q_{s-1,(s-1) / 2+1} & \cdots & q_{s-2, s} & q_{s-2,(s-1) / 2+1} & \cdots & q_{s-2, s}
\end{array}\right]^{T}
$$

if $s$ is odd or

$$
q_{p a r}=\left[\begin{array}{llllll}
q_{s-1, s / 2+1} & \cdots & q_{s-1, s} & q_{s-2, s / 2} & \cdots & q_{s-2, s}
\end{array}\right]^{T}
$$

if $s$ is even, are computed by maximizing the area of the intersection of the region of the absolute stability of the polynomial $q(w, z)$ with the negative half plane. This area function area $\left(q_{\text {par }}\right)$ is computed by numerical integration in polar coordinates as explained in [14, 23]. This leads to the following minimization problem

$$
f\left(q_{\text {par }}\right)=-\operatorname{area}\left(q_{\text {par }}\right) \longrightarrow \min ,
$$

which is then solved using fminsearch from Matlab. For $p=s-1=5$ this polynomial in Matlab rational format (format rat) takes the form

$$
\begin{aligned}
& q_{s-1}(z)=1+\frac{1013}{1332} z+\frac{391}{862} z^{2}+\frac{105}{523} z^{3}+\frac{183}{4187} z^{4}+\frac{81}{18349} z^{5}+\frac{5}{29738} z^{6}, \\
& q_{s-2}(z)=-\frac{319}{1332} z-\frac{685}{2396} z^{2}-\frac{295}{2234} z^{3}-\frac{481}{17829} z^{4}-\frac{81}{31859} z^{5}-\frac{37}{406296} z^{6},
\end{aligned}
$$


and its area of the region of absolute stability is 45.96. For $p=s-1=6$ this polynomial takes the form

$$
\begin{aligned}
q_{s-1}(z)= & 1+\frac{851}{810} z+\frac{550}{1229} z^{2}+\frac{353}{2200} z^{3}+\frac{415}{8802} z^{4}+\frac{89}{10370} z^{5} \\
& +\frac{146}{180747} z^{6}+\frac{3}{99656} z^{7} \\
q_{s-2}(z)= & \frac{250}{4939} z-\frac{138}{74027} z^{2}-\frac{408}{12221} z^{3}-\frac{207}{11168} z^{4}-\frac{50}{12459} z^{5} \\
& -\frac{72}{183931} z^{6}-\frac{2}{138485} z^{7},
\end{aligned}
$$

and its area of the region of absolute stability is 50.90. For $p=s-1=7$ this polynomial in Matlab rational format takes the form

$$
\begin{aligned}
q_{s-1}(z)= & 1+\frac{1705}{1751} z+\frac{729}{1421} z^{2}+\frac{699}{4205} z^{3}+\frac{537}{13301} z^{4}+\frac{111}{12851} z^{5}+\frac{41}{28217} z^{6} \\
& +\frac{19}{130733} z^{7}+\frac{2}{330385} z^{8} \\
q_{s-2}(z)= & -\frac{77}{2931} z-\frac{89}{6716} z^{2}-\frac{22}{39821} z^{3}+\frac{25}{62304} z^{4}-\frac{6}{45311} z^{5}-\frac{1}{36037} z^{6} \\
& +\frac{1}{750761} z^{7}+\frac{1}{3461764} z^{8},
\end{aligned}
$$

and its area of the region of absolute stability is 47.29 .

We would like to add here that for $p \geq 5$, it was hard to find quadratic polynomials with large stability areas, and we needed to choose a suitable starting guess for the fminsearch Matlab function. In particular, for $p=$ $s-1=5$ we have used as starting point

$$
q_{p a r}^{(0)}=\left[\begin{array}{llllllll}
0 & 0 & 0 & 0 & \frac{1}{4 !} & \frac{1}{5 !} & \frac{1}{6 !} & 0
\end{array}\right]^{T},
$$

which corresponds to the approximation of the same order to the exponential function $\exp (z)$. For $p=s-1=6$ and $p=s-1=7$, we have used the starting point

$$
q_{\text {par }}^{(0)}=\left[\begin{array}{lllllllll}
0 & 0 & 0 & 0 & 0 & \frac{1}{5 !} & \frac{1}{6 !} & \frac{1}{7 !} & \frac{\eta}{8 !}
\end{array}\right]^{T},
$$

with $\eta=0.62$ and $\eta=0.61$ respectively, which corresponds to the stability polynomial of the IRKS of the same order with maximum area.

\section{Search for Nordsieck Methods with Quadratic Stability}

In this section we describe the construction of Nordsieck methods (2.2) whose stability function $p(w, z)$ assumes the form

$$
p(w, z)=w^{s-2} q_{\text {opt }}(w, z),
$$


where $q_{\text {opt }}(w, z)$ are optimal quadratic polynomials which are computed by solving minimization problem (3.2). It can be verified that the stability function $p(w, z)$ of the method (2.2) with $r=s$ and $p=q=s-1$ assumes the form $p(w, z)=w^{s}-p_{s-1}(z) w^{s-1}+p_{s-2}(z) w^{s-2}+\cdots+(-1)^{s-1} p_{1}(z) w+(-1)^{s} p_{0}(z)$, where the polynomials $p_{s-1}(z), p_{s-2}(z), \ldots, p_{0}(z)$ are given by

$$
\begin{aligned}
p_{s-1}(z)= & 1+p_{s-1,1} z+p_{s-1,2} z^{2}+\cdots+p_{s-1, s} z^{s}, \\
p_{s-2}(z)= & p_{s-2,1} z+p_{s-2,2} z^{2}+\cdots+p_{s-2, s} z^{s} \\
& \cdots \\
p_{1}(z)= & p_{1,1} z+p_{1,2} z^{2}+\cdots+p_{1, s} z^{s} \\
p_{0}(z)= & p_{0,1} z+p_{0,2} z^{2}+\cdots+p_{0, s} z^{s} .
\end{aligned}
$$

Hence, to obtain methods with QS whose stability polynomial $p(w, z)$ takes the form (4.1) we have to enforce the conditions

$$
p_{j, k}=q_{j, k}, \quad j=s-1, s-2, k=1,2, \ldots, s,
$$

where $q_{j, k}$ are coefficients of the polynomials $q_{s-1}(z)$ and $q_{s-2}(z)$ and

$$
p_{j, k}=0, \quad j=0,1, \ldots, s-3, k=1,2, \ldots, s .
$$

This leads to the minimization problem

$$
f\left(\mathbf{A}, \mathbf{V}, \mathbf{b}_{s}\right) \longrightarrow \min ,
$$

where the objective function $f\left(\mathbf{A}, \mathbf{V}, \mathbf{b}_{s}\right)$ is defined by

$$
f\left(\mathbf{A}, \mathbf{V}, \mathbf{b}_{s}\right):=\sum_{j=s-1}^{s-2} \sum_{k=1}^{s}\left|p_{j, k}-q_{j, k}\right|^{2}+\sum_{j=0}^{s-3} \sum_{k=1}^{s}\left|p_{j k}\right|^{2} .
$$

To compute this objective function we need expressions for the coefficients $p_{j, k}$. It was demonstrated in $[8,19]$ using the approach based on Fourier series that these coefficients are given by

$$
p_{j k}=(-1)^{s-j} \frac{1}{N_{1} N_{2}} \sum_{\mu=0}^{N_{1}-1} \sum_{\nu=0}^{N_{2}-1} w_{\mu}^{-j} z_{\nu}^{-k} p\left(w_{\mu}, z_{\nu}\right),
$$

$j=0,1, \ldots, s-1, k=0,1, \ldots, s$, where

$$
\begin{aligned}
& w_{\mu}=\exp \left(-\frac{2 \pi \mu i}{N_{1}}\right), \quad \mu=0,1, \ldots, N_{1}-1, \\
& z_{\nu}=\exp \left(-\frac{2 \pi \nu i}{N_{2}}\right), \quad \nu=0,1, \ldots, N_{2}-1
\end{aligned}
$$

are complex numbers uniformly distributed on the unit circle, and $N_{1}$ and $N_{2}$ are sufficiently large integers. The efficient computation of these coefficients by fast Fourier transform technique is described in [13]. 
The minimization problem (4.4) with objective function defined by (4.5) was solved by applying the Levenberg-Marquardt variant of the Gauss-Newton method to this least squares problem. To ensure convergence to sufficiently small residual values a smooth damping strategy was employed as suggested in [25] and the Jacobian was computed by high-order numerical differentiation. The standard first order one-sided difference formula was replaced by a symmetric interpolation by a fourth degree polynomial. For smooth functions this provides very accurate derivatives at the cost of six function evaluations. In light of the complex dependence of the objective on the variables both exact and automatic differentiation are practically impossible.

Observe that the solutions to the minimization problem (4.4) for which the objective function $f\left(\mathbf{A}, \mathbf{V}, \mathbf{b}_{s}\right)$ is equal to zero are also the solutions to (4.2) and (4.3).

The examples of methods found using the approach described in this section are presented in Section 5. We also present the corresponding regions of absolute stability of these methods and compare them with stability regions of approximation to the exponential function $\exp (z)$ of the same order and of the GLMs with IRKS of the same order.

\section{Examples of Methods}

In this section we present examples of methods with QS obtained using the minimization algorithm described in Section 4. We also compare their stability regions with stability regions of approximation of the same order to the exponential function $\exp (z)$ as well as with Nordsieck method (2.2) of order $p=s-1$ with IRKS. We list only the coefficient matrices $\mathbf{A}, \mathbf{V}$ and the last column $\mathbf{b}_{s}$ of the matrix $\mathbf{B}$. These coefficients are presented in the Matlab rational format. In actual implementation of these methods the coefficients matrix $\mathbf{U}$ should be computed to a full precision from the representation formula $\mathbf{U}=\mathbf{C}-\mathbf{A C K}$, and the first $s-1$ columns $\tilde{B}$ of the matrix $\mathbf{B}$ should be computed to a full precision from the representation formula (2.5) discussed in Section 2.

\section{$5.1 s=r=6, p=q=5$}

We have found methods with coefficients $\mathbf{A}, \mathbf{V}$ and $\mathbf{b}_{s}$ for which the objective function $f\left(\mathbf{A}, \mathbf{V}, \mathbf{b}_{s}\right)$ was in the range of $10^{-7}-10^{-10}$. For methods for which $f\left(\mathbf{A}, \mathbf{V}, \mathbf{b}_{s}\right)$ was about $10^{-7}$ the stability regions are quite irregular and differ markedly from stability region of optimal quadratic polynomial $q_{\text {opt }}(w, z)$ computed in Section 3. For methods for which $f\left(\mathbf{A}, \mathbf{V}, \mathbf{b}_{s}\right)$ was about $10^{-8}$ or less the stability region of the method could not be distinguished on the computer screen from stability region of $q_{o p t}(w, z)$.

We have plotted in Fig. 1 and in Fig. 2 stability regions of the methods with $f\left(\mathbf{A}, \mathbf{V}, \mathbf{b}_{s}\right)$ equal to $7 \cdot 10^{-7}$ and $9 \cdot 10^{-10}$, respectively, and stability region of $q_{\text {opt }}(w, z)$ corresponding to $p=s-1=5$. The coefficients of the method for 


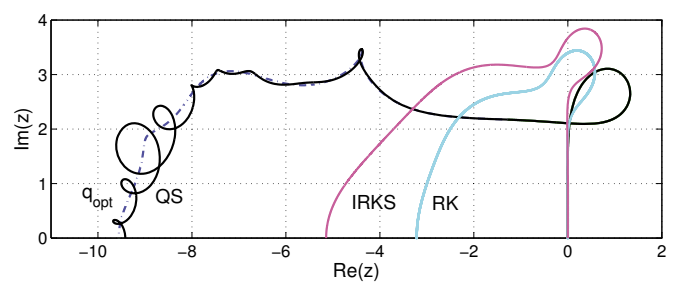

Figure 1. Stability regions of approximation of order $p=5$ to $\exp (z)$, of GLM with IRKS corresponding to $\eta=3 / 5$, and of GLMs of order $p=s-1=5$ with QS with residual $=7 \cdot 10^{-7}$. Stability region of optimal quadratic polynomial $q_{o p t}$ is plotted by dashed line.

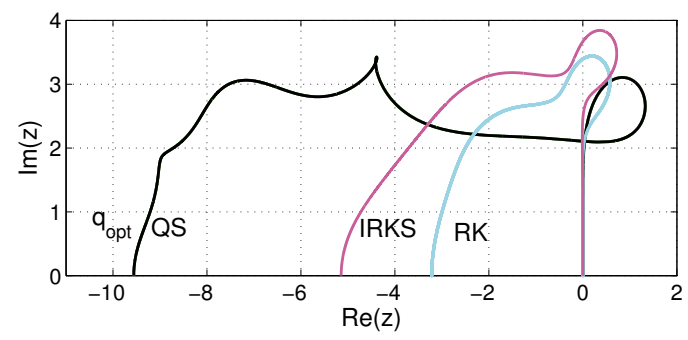

Figure 2. Stability regions of approximation of order $p=5$ to $\exp (z)$, of GLM with IRKS corresponding to $\eta=3 / 5$, and of GLMs of order $p=s-1=5$ with QS with residual $=9 \cdot 10^{-10}$. Stability region of optimal quadratic polynomial $q_{\text {opt }}$ cannot be distinguished from stability region of GLM with QS.

which $f\left(\mathbf{A}, \mathbf{V}, \mathbf{b}_{s}\right)=9 \cdot 10^{-10}$ are $\mathbf{c}=\left[\begin{array}{llllll}0 & \frac{1}{5} & \frac{2}{5} & \frac{3}{5} & \frac{4}{5} & 1\end{array}\right]^{T}$,

$$
\begin{gathered}
\mathbf{A}=\left[\begin{array}{cccccc}
0 & 0 & 0 & 0 & 0 & 0 \\
\frac{484}{1413} & 0 & 0 & 0 & 0 & 0 \\
\frac{339}{1001} & \frac{320}{1401} & 0 & 0 & 0 & 0 \\
\frac{541}{1504} & -\frac{241}{2590} & \frac{411}{1039} & 0 & 0 & 0 \\
\frac{303}{719} & -\frac{788}{1091} & \frac{1244}{1947} & \frac{675}{2351} & 0 & 0 \\
\frac{557}{697} & \frac{622}{3809} & \frac{243}{646} & \frac{359}{1256} & \frac{1073}{4861} & 0
\end{array}\right], \\
\mathbf{V}=\left[\begin{array}{cccccc}
1 & \frac{517}{16821} & \frac{90}{1643} & \frac{94}{6201} & \frac{104}{25257} & \frac{20}{26753} \\
0 & 0 & -\frac{115}{251467} & -\frac{77}{14673} & -\frac{79}{9456} & \frac{48}{31057} \\
0 & 0 & 0 & -\frac{43}{15540} & -\frac{103}{8944} & \frac{58}{11891} \\
0 & 0 & 0 & 0 & -\frac{298}{3897} & \frac{89}{4543} \\
0 & 0 & 0 & 0 & 0 & \frac{1}{1453706} \\
0 & 0 & 0 & 0 & 0 & 0
\end{array}\right], \\
\mathbf{b}_{s}=\left[\begin{array}{llllll}
323 \\
\frac{3268}{3768} & \frac{268}{2157} & \frac{1040}{1249} & \frac{493}{881} & -\frac{1051}{1712} & \frac{326}{1355}
\end{array}\right]^{T} .
\end{gathered}
$$


The error constant of this method is 0.0023. For comparison, we have also plotted on the same figure stability region of approximation of the same order to $\exp (z)$ which corresponds to the polynomial $R(z, \eta)$ given by $(2.8)$ with $\eta=0$ and of GLM with IRKS with stability function given by $(2.7)$ with $R(z, \eta)$ given by (2.8) for $\eta=3 / 5$, with the error constant equal to $1 / 360$. It can be verified that this value of $\eta$ maximizes the area of the stability region of IRKS method of order $p=s-1=5$.

\section{2 $s=r=7, p=q=6$}

We have found methods with coefficients $\mathbf{A}, \mathbf{V}$ and $\mathbf{b}_{s}$ for which the objective function $f\left(\mathbf{A}, \mathbf{V}, \mathbf{b}_{s}\right)$ was in the range of $10^{-7}-10^{-9}$. Similarly as in the case $p=s-1=6$, when the objective function $f\left(\mathbf{A}, \mathbf{V}, \mathbf{b}_{s}\right)$ was about $10^{-8}$ or less, the stability region of the resulting method could not be distinguished on the computer screen from stability region of the optimal quadratic polynomial $q_{\text {opt }}(w, z)$.

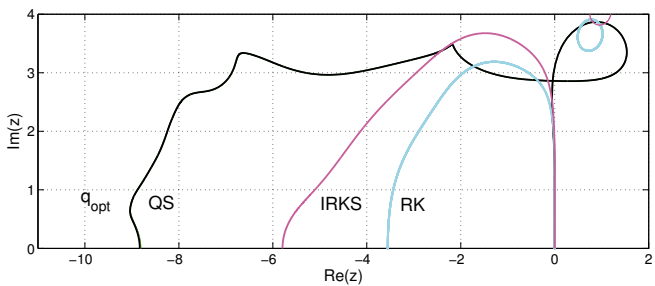

Figure 3. Stability regions of approximation of order $p=6$ to $\exp (z)$, of GLM with IRKS corresponding to $\eta=0.59$, and of GLM with $s=7$ and $p=6$ with QS with residual $=4 \cdot 10^{-9}$. Stability region of optimal quadratic polynomial $q_{o p t}$ cannot be distinguished from stability region of GLM with QS.

We have plotted in Fig. 3 the stability region of the method corresponding to $f\left(\mathbf{A}, \mathbf{V}, \mathbf{b}_{s}\right)$ equal to $4 \cdot 10^{-9}$ and stability region of $q_{o p t}(w, z)$ corresponding to $p=s-1=6$, given in Section 3. For comparison, we have also plotted the stability region of the approximation of the same order to $\exp (z)$. The coefficients of the method for which res $=4 \cdot 10^{-9}$ are $\mathbf{c}=\left[\begin{array}{lllllll}0 & \frac{1}{6} & \frac{1}{3} & \frac{1}{2} & \frac{2}{3} & \frac{5}{6} & 1\end{array}\right]^{T}$,

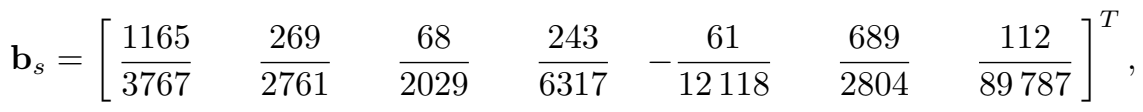

$A=\left[\begin{array}{ccccccc}0 & 0 & 0 & 0 & 0 & 0 & 0 \\ \frac{585}{4144} & 0 & 0 & 0 & 0 & 0 & 0 \\ \frac{1159}{12823} & \frac{2623}{13184} & 0 & 0 & 0 & 0 & 0 \\ \frac{206}{5273} & \frac{561}{2564} & \frac{227}{1216} & 0 & 0 & 0 & 0 \\ -\frac{230}{6781} & \frac{240}{761} & \frac{229}{2092} & \frac{549}{2546} & 0 & 0 & 0 \\ -\frac{203}{2554} & \frac{353}{1220} & \frac{1025}{4081} & \frac{172}{2261} & \frac{1369}{5756} & 0 & 0 \\ -\frac{187}{4478} & \frac{799}{2778} & \frac{724}{3703} & -\frac{174}{24439} & \frac{27}{6128} & \frac{282}{779} & 0\end{array}\right]$,




$$
V=\left[\begin{array}{ccccccc}
1 & \frac{109}{1948} & \frac{386}{7121} & \frac{87}{2026} & \frac{124}{13351} & -\frac{43}{44499} & -\frac{43}{49135} \\
0 & 0 & \frac{382}{3919} & \frac{456}{9871} & -\frac{163}{3084} & -\frac{37}{14932} & -\frac{141}{27967} \\
0 & 0 & 0 & \frac{182}{2007} & \frac{1605}{6526} & \frac{323}{13496} & \frac{41}{4098} \\
0 & 0 & 0 & 0 & -\frac{647}{20819} & \frac{97}{6504} & -\frac{40}{30623} \\
0 & 0 & 0 & 0 & 0 & -\frac{191}{18378} & -\frac{49}{182268} \\
0 & 0 & 0 & 0 & 0 & 0 & -\frac{53}{84355} \\
0 & 0 & 0 & 0 & 0 & 0 & 0
\end{array}\right] .
$$

The error constant of this method is $3.2287 \cdot 10^{-4}$. The GLM with IRKS with maximum area has stability polynomial $(2.7)$ with $R(z, \eta)$ given by $(2.8)$ and $\eta=0.59$, and the error constant is equal to $8.13 \cdot 10^{-5}$.

$5.3 s=r=8, p=q=7$

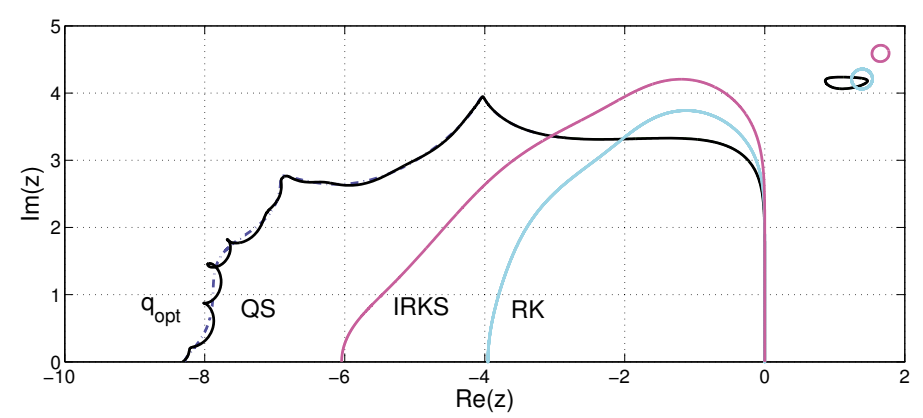

Figure 4. Stability regions of approximation of order $p=7$ to $\exp (z)$, of GLM with IRKS corresponding to $\eta=0.61$, of GLM with $s=8$ and $p=7$ with QS with residual $1.03 \cdot 10^{-7}$, and of optimal quadratic polynomial $q_{\text {opt }}$. Observe that the stability region of $q_{o p t}$ is quite close to stability region of GLM with QS.

We have found methods with coefficients $\mathbf{A}, \mathbf{V}$ and $\mathbf{b}_{s}$ for which the objective function $f\left(\mathbf{A}, \mathbf{V}, \mathbf{b}_{s}\right)$ was in the range of $10^{-6}-10^{-7}$. When the objective function $f\left(\mathbf{A}, \mathbf{V}, \mathbf{b}_{s}\right)$ was about $10^{-7}$, the stability region of the resulting method was very close to the stability region of the optimal quadratic polynomial $q_{\text {opt }}(w, z)$. We have plotted in Fig. 4 the stability region of the method corresponding to $f\left(\mathbf{A}, \mathbf{V}, \mathbf{b}_{s}\right)$ equal to $1.03 \cdot 10^{-7}$ and stability region of optimal quadratic polynomial $q_{\text {opt }}(w, z)$ corresponding to $p=s-1=7$ which is given in Section 3. The coefficients of the method for which $1.03 \cdot 10^{-7}$ are

$$
\begin{aligned}
& \mathbf{c}=\left[\begin{array}{llllllll}
0 & \frac{1}{7} & \frac{2}{7} & \frac{3}{7} & \frac{4}{7} & \frac{5}{7} & \frac{6}{7} & 1
\end{array}\right]^{T}, \\
& \mathbf{b}_{s}=\left[\begin{array}{lllllllll}
\frac{865}{1206} & -\frac{497}{4853} & -\frac{219}{1639} & -\frac{332}{5473} & \frac{310}{1349} & \frac{97}{11454} & -\frac{358}{5297} & -\frac{410}{3953}
\end{array}\right]^{T},
\end{aligned}
$$




$$
A=\left[\begin{array}{cccccccc}
0 & 0 & 0 & 0 & 0 & 0 & 0 & 0 \\
\frac{189}{1504} & 0 & 0 & 0 & 0 & 0 & 0 & 0 \\
\frac{379}{4434} & \frac{615}{3662} & 0 & 0 & 0 & 0 & 0 & 0 \\
\frac{181}{3915} & \frac{466}{2685} & \frac{476}{2913} & 0 & 0 & 0 & 0 & 0 \\
\frac{73}{5835} & \frac{283}{1614} & \frac{355}{2176} & \frac{281}{1714} & 0 & 0 & 0 & 0 \\
-\frac{166}{19269} & \frac{247}{1499} & \frac{157}{984} & \frac{577}{3350} & \frac{849}{5285} & 0 & 0 & 0 \\
-\frac{127}{26297} & \frac{692}{5835} & \frac{1373}{8299} & \frac{408}{2213} & \frac{151}{944} & \frac{335}{2086} & 0 & 0 \\
\frac{229}{3234} & \frac{398}{2691} & -\frac{137}{1184} & -\frac{300}{1667} & \frac{516}{2129} & -\frac{295}{2008} & \frac{129}{223} & 0
\end{array}\right],
$$

The error constant of this method is equal to $3.5 \cdot 10^{-5}$. For comparison, we have also plotted in Fig. 4 the stability region of the approximation of the same order to $\exp (z)$ and of the GLM with IRKS with stability polynomial (2.7) with $R(z, \eta)$ given by $(2.8)$ and $\eta=0.61$, with error constant $9.67 \cdot 10^{-6}$. It can be verified that this value of $\eta$ maximizes the area of stability region of IRKS method of order $p=s-1=7$.

\section{Concluding remarks}

This paper continues search for explicit GLMs in Nordsieck form with large regions of absolute stability. This search for methods of order up to $p=4$ was initiated in [13] and it was based on minimizing the negative are of the intersection of the region of absolute stability with a negative half plane. This was accomplished with the aid of subroutines fminsearch and fsolve from Matlab. For higher orders $(p \geq 5)$ the approach based on fminsearch and fsolve does not lead to sufficient accuracy and in this paper we used stateof-the art optimization software based on Levenberg-Marquardt algorithm to accomplish this goal for $p=5, p=6$, and $p=7$. In our approach we first construct quadratic polynomials $q_{o p t}(w, z)$ with large regions of absolute stability, and then use optimization software to find methods for which stability function $p(w, z)$ takes the form $p(w, z)=w^{s-2} q_{o p t}(w, z)$.

Future work in this area will be concerned with the construction of highly stable implicit GLMs in Nordsieck form and with implementation of both explicit and implicit methods in a variable step variable order software for nonstiff 
and stiff differential systems. The tools to accomplish this are different from the tools employed in this paper and this work will be reported elsewhere.

\section{Acknowledgements}

The results reported in this paper were obtained during the visit of the first author to the Arizona State University in October and November of 2010. This author wish to express her gratitude to the Department of Mathematics for hospitality during this visit.

\section{References}

[1] E.L. Allgower and K. Georg. Numerical Continuation Methods: An Introduction, volume 13 of Springer Ser. Comput. Math. Springer-Verlag, Berlin, 1990. ISBN 3-540-12760-7.

[2] E.L. Allgower and K. Georg. Continuation and path following. In Acta Numerica, 1993, Acta Numer., pp. 1-64. Cambridge Univ. Press, Cambridge, 1993.

[3] Z. Bartoszewski and Z. Jackiewicz. Construction of two-step Runge-Kutta methods of high order for ordinary differential equations. Numer. Algorithms, 18(1):51-70, 1998. http://dx.doi.org/10.1023/A:1019157029031.

[4] M. Braś. Nordsieck methods with inherent quadratic stability. Math. Model. Anal., 16(1):82-96, 2011. http://dx.doi.org/10.3846/13926292.2011.560617.

[5] J. C. Butcher and Z. Jackiewicz. Construction of diagonally implicit general linear methods of type 1 and 2 for ordinary differential equations. Appl. Numer. Math., 21(4):385-415, 1996.

[6] J.C. Butcher. Diagonally-implicit multi-stage integration methods. Appl. Numer. Math., 11(5):347-363, 1993. http://dx.doi.org/10.1016/0168-9274(93)90059-Z.

[7] J.C. Butcher. Numerical Methods for Ordinary Differential Equations. John Wiley \& Sons Ltd., Chichester, 2003. ISBN 0-471-96758-0.

[8] J.C. Butcher and Z. Jackiewicz. Construction of high order diagonally implicit multistage integration methods for ordinary differential equations. Appl. Numer. Math., 27(1):1-12, 1998.

[9] J.C. Butcher and Z. Jackiewicz. Construction of general linear methods with Runge-Kutta stability properties. Numer. Algorithms, 36(1):53-72, 2004. http://dx.doi.org/10.1023/B:NUMA.0000027738.54515.50.

[10] J.C. Butcher, Z. Jackiewicz and H.D. Mittelmann. A nonlinear optimization approach to the construction of general linear methods of high order. J. Comput. Appl. Math., 81(2):181-196, 1997. http://dx.doi.org/10.1016/S0377-0427(97)00039-3.

[11] J.C. Butcher, Z. Jackiewicz and W.M. Wright. Error propagation of general linear methods for ordinary differential equations. J. Complexity, 23(4-6):560580, 2007. http://dx.doi.org/10.1016/j.jco.2007.01.009.

[12] J.C. Butcher and W.M. Wright. The construction of practical general linear methods. BIT, 43(4):695-721, 2003. http://dx.doi.org/10.1023/B:BITN.0000009952.71388.23. 
[13] A. Cardone and Z. Jackiewicz. Explicit Nordsieck methods with quadratic stability. Numer. Algorithms. http://dx.doi.org/10.1007/s11075-011-9509-y. to appear

[14] J. Chollom and Z. Jackiewicz. Construction of two-step Runge-Kutta methods with large regions of absolute stability. J. Comput. Appl. Math., 157(1):125-137, 2003. http://dx.doi.org/10.1016/S0377-0427(03)00382-0.

[15] D. Conte, R. D'Ambrosio and Z. Jackiewicz. Two-step Runge-Kutta methods with quadratic stability functions. J. Sci. Comput., 44(2):191-218, 2010. http://dx.doi.org/10.1007/s10915-010-9378-x.

[16] J.E. Dennis, D.M. Gay and R.E. Welsch. An adaptive nonlinear least-squares algorithm. ACM Trans. Math. Software, 7:348-368, 1981.

http://dx.doi.org/10.1145/355958.355965.

[17] J.E. Dennis, D.M. Gay and R.E. Welsch. ALGORITHM 573: NL2SOL-An adaptive nonlinear least-squares algorithm. ACM Trans. Math. Software, 7:369383, 1981. http://dx.doi.org/10.1145/355958.355966.

[18] D.C. Hanselman and B.L. Littlefield. Mastering MATLAB \%. Prentice-Hall Inc., Upper Saddle River, NJ, 2004.

[19] Z. Jackiewicz. General Linear Methods for Ordinary Differential Equations. John Wiley \& Sons Inc., Hoboken, NJ, 2009. ISBN 978-0-470-40855-1.

[20] Z. Jackiewicz and H.D. Mittelmann. Exploiting structure in the construction of DIMSIMs. J. Comput. Appl. Math., 107(2):233-239, 1999. http://dx.doi.org/10.1016/S0377-0427(99)00091-6.

[21] Z. Jackiewicz and S. Tracogna. A general class of two-step Runge-Kutta methods for ordinary differential equations. SIAM J. Numer. Anal., 32(5):1390-1427, 1995. http://dx.doi.org/10.1137/0732064.

[22] Z. Jackiewicz and S. Tracogna. Variable stepsize continuous two-step RungeKutta methods for ordinary differential equations. Numer. Algorithms, 12(34):347-368, 1996. http://dx.doi.org/10.1007/BF02142812.

[23] Z. Jackiewicz and J.H. Verner. Derivation and implementation of two-step Runge-Kutta pairs. Japan J. Indust. Appl. Math., 19(2):227-248, 2002. http://dx.doi.org/10.1007/BF03167454.

[24] J.E. Dennis Jr. and R.B. Schnabel. Numerical methods for unconstrained optimization and nonlinear equations. Prentice-Hall Series in Computational Mathematics. Prentice-Hall Inc., Englewood Cliffs, NJ, 1983. ISBN 0-13-627216-9.

[25] H.B. Nielsen. Damping parameter in Marquardt's method. Technical Report IMM-REP-1999-05, Informatics and Mathematical Modelling, Technical University of Denmark, DTU, DK-2800 Kgs. Lyngby, 1999. Available from Internet: http://www2.imm.dtu.dk/pubdb/p.php?648.

[26] W.C. Rheinboldt and J.V. Burkardt. Algorithm 596: A program for a locally parametrized continuation process. ACM Trans. Math. Software, 9(2):236-241, 1983. http://dx.doi.org/10.1145/357456.357461.

[27] W.C. Rheinboldt and J.V. Burkardt. A locally parameterized continuation process. ACM Trans. Math. Software, 9:215-235, 1983.

http://dx.doi.org/10.1145/357456.357460.

[28] S. Tracogna. A general class of two-step Runge-Kutta methods for ordinary differential equations. Doctoral thesis, Arizona State University, Arizona, 1996. 
[29] L.T. Watson, S.C. Billups and A.P. Morgan. Algorithm 652: HOMPACK: a suite of codes for globally convergent homotopy algorithms. ACM Trans. Math. Software, 13:281-310, 1987. http://dx.doi.org/10.1145/29380.214343.

[30] W.M. Wright. Explicit general linear methods with inherent Runge-Kutta stability. Numer. Algorithms, 31(1-4):381-399, 2002. http://dx.doi.org/10.1023/A:1021195804379.

[31] W.M. Wright. General linear methods with inherent Runge-Kutta stability. Doctoral thesis, The University of Auckland, New Zealand, 2002. 